\title{
Low diversity of the gut microbiota in infants with atopic eczema
}

Thomas Abrahamsson, Hedvig E Jakobsson, Anders F Andersson, Bengt Bjorksten, Lars Engstrand and Maria Jenmalm

\section{Linköping University Post Print}

N.B.: When citing this work, cite the original article.

Original Publication:

Thomas Abrahamsson, Hedvig E Jakobsson, Anders F Andersson, Bengt Bjorksten, Lars Engstrand and Maria Jenmalm, Low diversity of the gut microbiota in infants with atopic eczema, 2012, Journal of Allergy and Clinical Immunology, (129), 2, 434-440.

http://dx.doi.org/10.1016/j.jaci.2011.10.025

Copyright: Elsevier

http://www.elsevier.com/

Postprint available at: Linköping University Electronic Press

http://urn.kb.se/resolve?urn=urn:nbn:se:liu:diva-75901 
Thomas R Abrahamsson, MD, $\mathbf{P h D}^{1}$

Hedvig E Jakobsson, MSc ${ }^{2,3}$

Anders F Andersson, $\mathbf{P h D}^{4}$

Bengt Björkstén, $\mathrm{MD}, \mathrm{PhD}^{5}$

Lars Engstrand, $\mathrm{MD}, \mathbf{P h D}^{2,3}$

Maria C Jenmalm, $\mathbf{P h D}^{1,6}$

1. Department of Clinical and Experimental Medicine, Division of Pediatrics, Linköping University, Sweden

2. Department of Preparedness, Swedish Institute for Communicable Disease Control, Solna, Sweden

3. Department of Microbiology, Tumor and Cell Biology, Karolinska Institutet, Stockholm, Sweden

4. Science for Life Laboratory, School of Biotechnology, KTH Royal Institute of Technology, Stockholm, Sweden

5. Institute of Environmental Medicine, Karolinska Institutet, Stockholm, and School of Health and Medical Sciences, Örebro University Sweden

6. Department of Clinical and Experimental Medicine, Unit of Autoimmunity and Immune Regulation, Division of Clinical Immunology, Linköping University, Sweden 
Correspondence to: Thomas Abrahamsson

Division of Paediatrics

SE-581 85 Linköping,Sweden

Phone: +46-(10)-1030000

Fax: +46-(13)-148265.

33 Supported by grants from BioGaia AB, Stockholm, Sweden, the Ekhaga Foundation, the

34 Heart and Lung foundation, the Research Council for the South-East Sweden (grant No.

35 F2000-106), The Olle Engqvist Foundation, the Swedish Asthma and Allergy Association,

36 the Swedish Research Council,the University Hospital of Linköping, the Söderberg

37 Foundation, the Vårdal Foundation for Health Care Science and Allergy Research, Sweden. 
42

43

44

45

\section{ABSTRACT}

Background: It is debated whether a low total diversity of the gut microbiota in early childhood is more important than altered prevalence of particular bacterial species for the increasing incidence of allergic disease. The advent of powerful, cultivation-free, molecular methods makes it possible to characterize the total microbiome down to the genus level in large cohorts.

Objective: To assess microbial diversity and characterize the dominant bacteria in stool during the first year of life in relation to atopic eczema development.

Methods: The microbial diversity and composition was analyzed with barcoded 16S rDNA 454-pyrosequencing in stool samples at one week, one month and 12 months of age in 20 infants developing IgE-associated eczema and 20 infants without any allergic manifestation until two years of age. (ClinicalTrials.gov ID NCT01285830)

Results: Infants who developed IgE-associated eczema had a lower diversity of the total microbiota at one month $(\mathrm{p}=0.004)$ and lower diversity of the bacterial phyla Bacteriodetes and the genus Bacteroides at one month $(\mathrm{p}=0.02$ and $\mathrm{p}=0.01)$ and Proteobacteria at 12 months of age $(\mathrm{p}=0.02)$. The microbiota was less uniform at one month than 12 months of age, with a high inter-individual variability. At 12 months, when the microbiota had stabilized, Proteobacteria, comprising gram negatives, were more abundant in infants without allergic manifestation (Edge $\mathrm{R}$ test $\mathrm{p}=0.008, \mathrm{q}=0.02)$.

Conclusion: Low intestinal microbial diversity during the first month of life was associated with subsequent atopic eczema.

Key message: Low microbial diversity early in life is associated with increased risk for allergic disease. 
67 With a novel powerful non-cultivation based method, infants who developed atopic eczema

68 were shown to have a low intestinal microbial diversity during the first month of life, in

69 particular low diversity of Bacteroidetes and Protebacteria.

$71 \quad$ Key words

72 Allergic disease; Bacteroides; diversity; eczema; hygiene hypothesis; infant; microbiota;

73 molecular microbiology; pyrosequencing; Sutterella

74

75 Abbreviations

76 BLAST: Basic Local Alignment Search Tool

77 CV: Coefficient of variance

78 DGGE: Denaturating gradient gel electrophoresis

79 Edge R: Empirical analysis of digital gene expression in $\mathrm{R}$

80 FISH: Fluorescent in situ hybridization

81 LPS: Lipopolysaccharides

82 OTU: Operational Taxonomic Unit

83 RDP: Ribosomal Database Project

84 SPT: skin prick test

85 T-RFLP: Terminal restriction fragment length polymorphism 


\section{INTRODUCTION}

It is debated whether low diversity of the gut microbiota in infancy is more important than the prevalence of specific bacterial taxa when trying to explain why the prevalence of allergic disease is increasing in affluent countries. Initially, several studies employing conventional cultivation or fluorescent in situ hybridization (FISH) reported differences in the intestinal microbiota at a species level between allergic and non-allergic children. ${ }^{1-3}$ Allergic infants were colonized less often with Bacteroides and bifidobacteria, ${ }^{1,2}$ more often with Staphylococcus aureus, ${ }^{2}$ and they had lower ratio of bifidobacteria to clostridia. ${ }^{3}$ However, there have been contradictory results in more recent studies. Two large European prospective studies did not confirm any relationship with any particular bacterial group. ${ }^{4,5}$

As an alternative explanation, it has been suggested that low diversity of intestinal microbiota would explain the increase of allergic disease in affluent societies. ${ }^{6,7}$ The underlying rationale is that the gut immune system reacts to exposure to new bacterial antigens and repeated exposure would enhance the development of immune regulation. Although this theory emerged more than a decade ago, ${ }^{8}$ there are still only few studies relating the diversity with allergy, likely due to methodology limitations. In three studies employing molecular techniques, terminal restriction fragment length polymorphism (T-RFLP) ${ }^{6}$ and denaturating gradient gel electrophoresis (DGGE) ${ }^{9,10}$, infants developing sensitization ${ }^{10}$ or eczema 69 were reported to have fewer peaks/bands than healthy ones. Yet, no specific microbes were identified with these molecular methods. Furthermore, the sensitivity of the methods appears to be low, since the median number of peaks/bands was much lower than the expected number of bacterial species. ${ }^{6,9,10}$

A new generation of powerful non-cultivation microbiology methods has now made it possible to analyze the total microbiota down to the genus level, even in large cohorts. ${ }^{11,12}$ 
112 Previously uncultivated bacteria can now be detected, and there is no need to decide what

113 bacteria to analyze in advance. Thus the assessment can be made unprejudiced. This will

114 allow more comprehensive knowledge of the intestinal microbiota and its impacts on the

115 immune system. We have employed barcoded 16S rRNA 454-pyrosequencing ${ }^{13}$ to assess the

116 microbial diversity and characterize the dominant bacteria in stool during the first year of life

117 in infants who either developed atopic eczema or did not have any allergic manifestation up to

118 two years of age.

119

120

121 
122

123

124

125

126

127

128

129

130

131

132

133

134

135

136

137

138

139

140

141

142

143

\section{Diagnostic criteria of atopic eczema}

145 Eczema was defined as a pruritic, chronic or chronically relapsing non-infectious dermatitis

146 with typical features and distribution. ${ }^{14}$ The diagnosis atopic eczema required that the infant 
147 with eczema also was sensitized. ${ }^{15}$ Infants were regarded as sensitized if they had at least one

148 positive SPT and/or detectable circulating allergen specific IgE antibodies. Skin prick tests

149 were done on the volar aspects of the forearm with egg white, fresh skimmed cow milk (lipid

150 concentration $0.5 \%$ ) and standardised cat, birch and timothy extracts (Soluprick ${ }^{\circledR}$, ALK,

151 Hørsholm, Denmark) at 6,12 and 24 months of age. Histamine hydrochloride $(10 \mathrm{mg} / \mathrm{ml})$ was

152 used as positive and albumin diluents as negative control. The test was regarded as positive if

153 the mean diameter of the wheal was $>3 \mathrm{~mm}$. Circulating IgE antibodies to egg white and

154 cow's milk were analysed at 6,12 , and 24 months of age in venous blood (UniCap ${ }^{\circledR}$

155 Pharmacia CAP System ${ }^{\mathrm{TM}}$, Pharmacia Diagnostics, Uppsala, Sweden). The cut off level was

$1560.35 \mathrm{kU} / \mathrm{L}$, according to the protocol of the manufacturer. In addition, circulating IgE to a

157 mixture of food allergens, including egg white, cow's milk, cod, wheat, peanut and soy bean,

158 was analysed at 6, 12 and 24 months of age (UniCap ${ }^{\circledR}$ Pharmacia CAP System ${ }^{\mathrm{TM}}$, fx5,

159 Pharmacia Diagnostics).

160

161 DNA extraction, 16S rRNA gene amplification, and sequencing

162 Extraction of bacterial DNA from the fecal samples and the 16S rRNA gene amplification

163 was made according to a previous publication ${ }^{13}$ with the following modifications; the primer

164 pair used, targeting the variable regions 3 and 4 of the 16S rRNA gene, were $341 \mathrm{f}$

165 5'CCTACGGGNGGCWGCAG with adaptor B and 805r

1665 'GACTACHVGGGTATCTAATCC with adaptor A ${ }^{16}$ and sample-specific sequence

167 barcodes consisting of five nucleotides. The barcodes contained no homopolymers and a pair

168 of barcodes differed in at least two positions. A negative PCR reaction without template was

169 also included for all primer pairs in each run. The PCR was run for 25 cycles. The PCR-

170 products with proximal lengths of $450 \mathrm{bp}$ were purified with AMPure beads (Becton

171 Dickinson, Franklin, USA) using a Magnet Particle Separator (Invitrogen, Carlsbad, CA, 
172 USA). The concentrations were measured by Qubit fluorometer (Invitrogen) CA), the quality

173 was assessed on a Bioanalyzer 2100 (Agilent, Santa Clara, USA), and the samples were

174 pooled together and amplified in PCR-mixture-in-oil emulsions and sequenced on different

175 lanes of a 2-lane PicoTiterPlate on a Genome Sequencer FLX system (Roche, Basel,

176 Switzerland) at the Royal Institute of Technology (KTH) in Stockholm.

177

178

179

180

181

182

183

184

185

186

187

188

189

190

191

192

193

194

195

\section{Sequence processing and taxonomic classification}

Sequence processing was carried out with the AmpliconNoise software package ${ }^{17}$ correcting for errors introduced in the PCR and pyrosequencing as well as removing chimeric sequences. Also, reads lacking a correct primer and/or having less than 360 successful pyrosequencing flows were removed. ${ }^{17}$ Denoised sequences were trimmed to $198 \mathrm{bp}$ after primer and barcode removal and clustered by complete linkage clustering into operational taxonomic units (OTUs) at the $97 \%$ similarity level using AmpliconNoise. ${ }^{17}$ Each denoised sequence, as well as the most abundant sequence for each OTU, was BLAST searched with default parameters against a local BLAST database comprising 836.814 near full-length bacterial 16S rRNA gene sequences from the Ribosomal Database Project (RDP) v. 10.10. ${ }^{18}$ The sequences inherited the taxonomic annotation (down to genus level) of the best scoring RDP hit fulfilling the criteria of $\geq 95 \%$ identity over an alignment of length $\geq 180 \mathrm{bp}$. If no such hit was found the sequence was classified as "no match". If multiple best hits were found and these had conflicting taxonomies, the most detailed level of consensus taxonomy was assigned to the OTU. After removal of pyrosequencing noise and chimeric sequences, 271 355 high quality, typically 198 bp long, sequence reads remained, with 1137-12909 reads per sample $($ mean $=2261)$. These corresponded to 3597 unique sequences and 1818 OTUs, clustered at $97 \%$ similarity level using complete linkage clustering. The majority (98\%) of 
reads was of clear bacterial origin and had an RDP relative within 95\% sequence similarity.

197 Statistics on number of sequences and OTUs are presented in Table E1 (online repository).

\section{Statistical analysis}

200 Statistical significance testing over- and under-representation of the bacterial lineages was 201 made at phylum, class, genus, and OTU (3\% dissimilarity) levels. Comparisons were made 202 using the Bioconductor $R$ package (Empirical analysis of digital gene expression in $\mathrm{R}$ ) EdgeR

$203^{19}$, and p-values were converted to False Discovery Rate values (q-values) to correct for 204 multiple testing. ${ }^{19}$ EdgeR is a statistical test that is designed for the analysis of replicated 205 count-based expression data. The Shannon diversity index was employed to measure the 206 biodiversity in samples. Briefly, it is a test that takes in account the number of species and the 207 evenness of the species, typically with a value between $1.5-3.5 .{ }^{20}$ It was calculated as $-\Sigma$ $208 \log \left(\mathrm{p}_{i}\right) \mathrm{p}_{i}$, where $\mathrm{p}_{i}$ denotes the frequency of OTU ${ }_{i}{ }^{21}$ and differences in this index were tested 209 with Mann-Whitney U-test in the R software (http://www.r-project.org/). Clustering of OTUs 210 was analyzed with Fast Unifrac (http://bmf2.colorado.edu/fastunifrac/) ${ }^{22}$ by calculating 211 weighted sample distances.

212 Repeated-measures ANOVA was employed in analyses of multiple longitudinal measures of 213 a specific phylum or genus in subjects in two different groups The $X^{2}$ test was employed for 214 categorical data, unless the expected frequency for any cell was less than five, when Fisher's 215 exact test was employed (SPSS 16.0, SPSS Inc, Chicago, IL, USA). 


\section{RESULTS}

Infants who developed atopic eczema, i.e. IgE-associated eczema, had a lower diversity of the total microbiota and the bacterial phylum Bacteriodetes and its genus Bacteroides at one month of age than infants who did not have any allergic manifestation during the two first years of life (Table II). The diversity of the phylum Proteobacteria, comprising Gram negative 223 bacteria, was also reduced in the atopic infants, significantly so at 12 months of age (Table II). Furthermore, these phyla and genera differed significantly between atopic and non-atopic infants with repeated-measures ANOVA including all sampling time points during the first year of life (one month, one week and 12 months: $p=0.049$ for the total microbiota, $p=0.04$ for Bacteroidetes, $\mathrm{p}=0.02$ for Bacteroides and $\mathrm{p}=0.02$ for Proteobacteria). Probiotic supplementation was a potential confounder. Even after exclusion of the probiotic-treated infants, however, several significant differences and some statistical tendencies were still observed. $(\mathrm{p}=0.03$ for the total microbiota, $\mathrm{p}=0.06$ for proteobacteria, $\mathrm{p}=0.096$ for Bacteroidetes, $\mathrm{p}=0.03$ for Bacteroides at 1 month, and $\mathrm{p}=0.06$ for Proteobacteria and $\mathrm{p}=0.01$ for Bacteroidetes and Bacteroides at 12 months, data not shown). Nine infants received antibiotics between two and twelve months. Excluding them did not affect the result at 12 234 months $(\mathrm{p}=0.02$ for Proteobacteria).

The relative abundance of the dominant bacterial phyla at various ages is displayed in Figure 1. During the first month of life there was a high inter-subject variability (Figure E1, online

238 repository) and no significant differences at the phylum level between infants who did and did 239 not develop atopic eczema. The relative abundance of Bacteroidetes, Proteobacteria and 240 Actinobacteria, the latter a phylum comprising bifidobacteria, which are associated with 241 breastfeeding, was high in both groups. At 12 months, however, these phyla had declined and 242 Firmicutes, comprising Gram positive aerobe and anaerobe bacteria, had become dominant 
resembling an adult microbiota pattern. At this age the relative abundance of Proteobacteria was lower (Edge $\mathrm{R}$ test $\mathrm{p}=0.008, \mathrm{q}=0.02)$ and Firmicutes tended to be higher (Edge $\mathrm{R}$ test $\mathrm{p}=0.06, \mathrm{q}=0.10)$ in atopic than non-atopic infants (Table III). Infants that have received antibiotics or probiotics did not differ significantly in relative abundance from those that have not (data not shown). Despite this, the differences in relative abundance between healthy and atopic infants were more significant if infants receiving antibiotics were excluded (Edge $R$ test $\mathrm{p}=0.01, \mathrm{q}=0.02$ for Firmicutes, $\mathrm{p}=0.005, \mathrm{q}=0.02$ for Proteobacteria and $\mathrm{p}=0.03, \mathrm{q}=0.05$ for Bacteroidetes at 12 months, data not shown). Excluding infants receiving probiotics did not affect the relative abundance significantly.

In order to compare our findings with previous reports, which often relate allergic disease with bacterial classes and genera rather than phyla, the relative abundance of the dominant bacterial classes and genera is presented in Table III. Since 144 genera were identified, pvalues were converted to False Discovery Rate values (q-values) in order to correct for multiple testing. Bifidobacterium, Bacteroides, Streptococcus, Enterococcus and sequences collectively classified to unclassified Enterobacteriaceae were the most abundant genera, especially during the first month of life. There was no significant difference between atopics and non-atopics for any of the dominant bacterial genera, except for Enterococcus and Peptostreptococcaceae Incertae Sedis, which were more abundant in atopic infants at 12 months of age. Among less abundant genera (relative abundance $<1 \%$ ), only a few differed significantly between atopic and non-atopic infants after correcting for multiple testing. The microaerophilic Gram negative Sutterella, belonging to the phylum Proteobacteria, was more abundant in the non-atopic infants both at one and 12 months of age (healthy vs. atopic, mean $\%$ [SD]: 0.2 [0.4] vs. 0.006 [0.02], $\mathrm{p}=0.008, \mathrm{q}=0.02$ at one month; 0.3 [0.5] vs. 0.2 [0.5], $\mathrm{p}=0.006, \mathrm{q}=0.02$ at 12 months). The Gram negative anaerobe Fusobacterium, belonging to the 
268 phylum Fusobacteria, was also more abundant in this group at 12 months of age (healthy vs.

269 atopic, mean \% [SD]: 0.01 [0.02] vs. 0.002 [0.009], $\mathrm{p}=0.006, \mathrm{q}=0.02)$. On the other hand, the 270 Gram positive anaerobes Eggerthella, belonging to Actinobacteria, and Coprobacillus,

271 belonging to Firmicutes, were more abundant in the atopic infants at 12 months (healthy vs.

272 atopic, mean \% [SD]: 0.1 [0.2] vs. 0.8 [1.0], $\mathrm{p}<0.001, \mathrm{q}=0.002$, and 0.01 [0.04] vs. 0.4 [0.09], $273 \mathrm{p}<0.001, \mathrm{q}<0.001$, respectively). The Gram positive anaerobe Peptoniphilus, belonging to 274 Firmicutes, was more abundant at one month of age in the atopic infants (healthy vs. atopic, 275 mean \% [SD]: 0 [0] vs. $0.002[0.006], \mathrm{p}=0.01, \mathrm{q}=0.03)$. 


\section{DISCUSSION}

279 Employing the new high-throughput 16S based molecular microbiology, we could confirm 280 and extend previous findings, that low intestinal diversity during the first month of life is 281 associated with an increased risk of subsequent atopic disease. ${ }^{6910}$ In contrast to previous 282 studies, we could also show that the differences in diversity and relative abundance were 283 attributed to specific bacterial phyla and genera, possibly because the sensitivity of our 284 analyses was higher than in previous diversity studies. ${ }^{6,9,10}$ At 12 months, the mean of 285 OTUs/sample were 69 in our study, as compared to 8.5 bands/sample (in DGGE) in a recent 286 the study by Bisgaard et al. ${ }^{10}$ It is noteworthy that the most important differences appeared 287 the first months of life, supporting the theory that factors influencing the early of maturation 288 of the immune system might be especially important for subsequent allergy development. ${ }^{23}$ 289 The study, however, did not clarify the debate whether a low total diversity of the gut microbiota in early childhood is more important than altered prevalence of particular bacterial species in allergy development. Total diversity was important, but the differences in diversity and relative abundance seemed to be defined to specific bacteria.

The low diversity of the phylum Bacteroidetes and its genus Bacteroides in infants

295 developing atopic eczema is consistent with previous studies, reporting low levels of these 296 bacteria to be associated both with allergic disease ${ }^{2}$ and factors associated with allergic 297 disease, such as a Western lifestyle ${ }^{11,12}$ and caesarean section. ${ }^{24}$ Bacteroides species have 298 also been demonstrated to have anti-inflammatory properties. Thus, Bacteroides fragilis 299 prevented the induction of colitis via suppression of the pro-inflammatory cytokines TNF and 300 IL-23 in an experimental colitis model ${ }^{25}$ and also mediated a conversion from CD4+ T cells 301 into IL-10 producing Foxp3 T regulatory cells during commensal colonization eliciting 302 mucosal tolerance in another mice model. ${ }^{26}$ Furthermore, Bacteroides thetaiotaomicron 
303

modulates the expression of a large quantity of genes involved in mucosal barrier reinforcement. ${ }^{27,28}$

Although our results indicate that the microbial diversity is more important than the colonization with any particular bacteria, one bacterial phylum, Proteobacteria, appeared to be less abundant in the atopic infants. This phylum comprises Gram negative bacteria, typically with endotoxin (LPS) incorporated in their cell wall. Endotoxin elicits a Th1 response via the innate immune system by enhancing IL-12 production from monocytes and dendritic cells, ${ }^{29}$ and low exposure to endotoxin has been associated with increased risk of atopic eczema. ${ }^{30}$ Also, the low allergy prevalence among children growing up in farms and less affluent countries has been attributed to high endotoxin exposure. ${ }^{31,32}$ Thus, a strong endotoxin exposure may downregulate atopy-promoting $\mathrm{Th} 2$ responses, possibly causing the negative association between atopic eczema and high abundance and diversity of Proteobacteria in the present study.

Previously, bifidobacteria and clostridia, especially Clostridium difficile, have been associated with allergic disease. ${ }^{3,33}$ None of these bacteria were related to allergic disease in this study. Neither was Clostridium a dominant bacterial genus. However, there were other genera within the phylum Firmicutes that were more abundant in the atopic than the non-atopic infants. Interestingly, Firmicutes-have been associated to other conditions related to a westernized lifestyle, such as obesity. ${ }^{12,34}$

Importantly, assessments of stool samples merely reflect luminal colonic microbiota and not necessarily the colonization of the small intestine, in which the major part of the gut immune system is situated. The higher oxygen content in the upper gut favors facultative bacteria such as streptococci and lactobacilli, ${ }^{35}$ which therefore might be more important than our results indicate. 
Abrahamsson

330 In conclusion, the results support the hypothesis that low microbial diversity early in life is

331 associated with an increased risk for allergic disease. The importance of bacteria belonging to

332 the phyla Bacteroidetes and Proteobacteria was corroborated, while the importance of other

333 bacteria previously associated with allergic disease, such as bifidobacteria and clostridia,

334 could not be confirmed.

335

\section{Acknowledgements}

337 We thank Mrs Lena Lindell, Mrs Elisabeth Andersson, Mrs Linnea Andersson and Mrs Eivor

338 Folkesson, Dr Göran Oldaeus and Dr Ted Jacobsson for their brilliant and enthusiastic work

339 guiding the families through the study and all the sampling procedures. We also thank Mrs

340 Anne-Marie Fornander for excellent technical assistance and Christopher Quince for assisting

341 with sequence noise removal. 
1. Björkstén B, Naaber P, Sepp E, Mikelsaar M. The intestinal microflora in allergic Estonian and Swedish 2-year-old children. Clinical and Experimental Allergy 1999; 29:1-5.

2. Watanebe S, Narisawa Y, Arase S, Okamatsu H, Ikenaga T, Tajiri Y, et al. Differences in fecal microflora between patients with atopic dermatitis and healthy control subjects. Journal of Allergy and Clinical Immunology 2003; 111:587-91.

3. Kalliomäki M, Kirjavainen PV, Eerola E, Kero P, Salminen SJ, Isolauri E. Distinct patterns of neonatal gut microflora in infants in whom atopy was and was not developing. Journal of Allergy and Clinical Immunology 2001; 107:129-34.

4. Adlerberth I, Strachan DP, Matricardi PM, Ahrné S, Orfei L, Åberg N, et al. Gut microbiota and development of atopic ezcema in 3 European birth cohorts. Journal of Allergy and Clinical Immunology 2007; 120:343-50.

5. Penders J, Stobberingh EE, Thijs C, Adams H, Vink C, van Ree R, et al. Molecular fingerprinting of the intestinal microbiota of infants in whom atopic eczema was or was not developing. Clin Exp Allergy 2006; 36:1602-8.

6. Wang M, Karlsson C, Olsson C, Adlerberth I, Wold AE, Strachan DP, et al. Reduced diversity in the early fecal microbiota of infants with atopic eczema. J Allergy Clin Immunol 2008; 121:129-34.

7. Adlerberth I, Carlsson B, de Man P, Jalil F, Khan SR, Larsson P, et al. Intestinal colonization with Enterobacteriaceae in Pakistani and Swedish hospital-delivered infants. Acta Paediatr Scand 1991; 80:602-10.

8. Holt PG. Postnatal maturation of immune competence during infancy and childhood. Pediatr Allergy Immunol 1995; 6:59-70.

9. Forno E, Onderdonk AB, McCracken J, Litonjua AA, Laskey D, Delaney ML, et al. Diversity of the gut microbiota and eczema in early life. Clin Mol Allergy 2008; 6:11.

10. Bisgaard H, Li N, Bonnelykke K, Chawes BL, Skov T, Paludan-Muller G, et al. Reduced diversity of the intestinal microbiota during infancy is associated with increased risk of allergic disease at school age. J Allergy Clin Immunol 2011; 128:646-52 e5.

11. Turnbaugh PJ, Hamady M, Yatsunenko T, Cantarel BL, Duncan A, Ley RE, et al. A core gut microbiome in obese and lean twins. Nature 2009; 457:480-4.

12. De Filippo C, Cavalieri D, Di Paola M, Ramazzotti M, Poullet JB, Massart S, et al. Impact of diet in shaping gut microbiota revealed by a comparative study in children from Europe and rural Africa. Proc Natl Acad Sci U S A 2010; 107:14691-6.

13. Andersson AF, Lindberg M, Jakobsson H, Backhed F, Nyren P, Engstrand L. Comparative analysis of human gut microbiota by barcoded pyrosequencing. PLoS One 2008; 3:e2836.

14. Abrahamsson TR, Jakobsson T, Böttcher MF, Fredrikson M, Jenmalm MC, Björkstén $\mathrm{B}$, et al. Probiotics in prevention of IgE-associated eczema: a double blind randomised placebo-controlled trial. Journal of Allergy and Clinical Immunology 2007; 119:117480.

15. Johansson S, Bieber T, Dahl R, Friedman P, Lanier B, Lockley R, et al. Revised nomenclature for allergy for global use: Report of the Nomenclature Review Committe of the World Allergy Organization, October 2003. Journal of Allergy and Clinical Immunology 2004; 113:832-6. 
16. Herlemann DP, Labrenz M, Jurgens K, Bertilsson S, Waniek JJ, Andersson AF.

Transitions in bacterial communities along the $2000 \mathrm{~km}$ salinity gradient of the Baltic Sea. ISME J.

17. Quince C, Lanzen A, Davenport RJ, Turnbaugh PJ. Removing noise from pyrosequenced amplicons. BMC Bioinformatics 2011; 12:38.

18. Maidak BL, Cole JR, Lilburn TG, Parker CT, Jr., Saxman PR, Stredwick JM, et al. The RDP (Ribosomal Database Project) continues. Nucleic Acids Res 2000; 28:173-4.

19. Robinson MD, McCarthy DJ, Smyth GK. edgeR: a Bioconductor package for differential expression analysis of digital gene expression data. Bioinformatics 2010; 26:139-40.

20. MacDonald GM. Biogeography: Introduction to Space, Time, and Life. 2003.

21. Hayek. Surveying natural populations; 1996.

22. Hamady M, Knight R. Microbial community profiling for human microbiome projects: Tools, techniques, and challenges. Genome Res 2009; 19:1141-52.

23. Prescott SL. Early origins of allergic disease: a review of processes and influences during early immune development. Curr Opin Allergy Clin Immunol 2003; 3:125-32.

24. Penders J, Thijs C, Vink C, Stelma FF, Snijders B, Kummeling I, et al. Factors influencing the composition of the intestinal microbiota in early infancy. Pediatrics 2006; 118:511-21.

25. Mazmanian SK, Round JL, Kasper DL. A microbial symbiosis factor prevents intestinal inflammatory disease. Nature 2008; 453:620-5.

26. Round JL, Mazmanian SK. Inducible Foxp3+ regulatory T-cell development by a commensal bacterium of the intestinal microbiota. Proc Natl Acad Sci U S A 2010; 107:12204-9.

27. Hooper LV, Wong MH, Thelin A, Hansson L, Falk PG, Gordon JI. Molecular analysis of commensal host-microbial relationships in the intestine. Science 2001; 291:881-4.

28. Freitas M, Axelsson LG, Cayuela C, Midtvedt T, Trugnan G. Indigenous microbes and their soluble factors differentially modulate intestinal glycosylation steps in vivo. Use of a "lectin assay" to survey in vivo glycosylation changes. Histochem Cell Biol 2005; 124:423-33.

29. Doreswamy V, Peden DB. Modulation of asthma by endotoxin. Clin Exp Allergy 2010; 41:9-19.

30. Gehring U, Bolte G, Borte M, Bischof W, Fahlbusch B, Wichmann HE, et al. Exposure to endotoxin decreases the risk of atopic eczema in infancy: A cohort study. Journal of Allergy and Clinical Immunology 2001; 108:847-54.

31. Riedler J, Braun-Fahrländer C, Eder W, Schreuer M, Waser M, Maisch S, et al. Exposure to farming in early life and development of asthma and allergy: a crosssectional survey. Lancet 2001; 358:1129-33.

32. Böttcher MF, Björkstén B, Gustafsson S, Voor T, Jenmalm MC. Endotoxin levels in Estonian and Swedish house dust and atopy in infancy. Clinical and Experimental Allergy 2003; 33:295-300.

33. Penders J, Thijs C, van den Brandt PA, Kummeling I, Snijders B, Stelma F, et al. Gut microbiota composition and development of atopic manifestations in infancy: the KOALA Birth Cohort Study. Gut 2007; 56:661-7.

34. Ley RE, Backhed F, Turnbaugh P, Lozupone CA, Knight RD, Gordon JI. Obesity alters gut microbial ecology. Proc Natl Acad Sci U S A 2005; 102:11070-5.

35. Hayashi H, Takahashi R, Nishi T, Sakamoto M, Benno Y. Molecular analysis of jejunal, ileal, caecal and recto-sigmoidal human colonic microbiota using 16S rRNA gene libraries and terminal restriction fragment length polymorphism. J Med Microbiol 2005; 54:1093-101. 
Abrahamsson

440

441

442 
444

445

446

447

448

449

450

451

452

453

454

455

456

457

458

459

460

461

462

463

464

465

466

467

468

469

470

471

472

473

TABLE I. Descriptive data of children included in the study.

\begin{tabular}{llll}
\hline & $\begin{array}{l}\text { Atopic eczema } \\
\%(\mathrm{n})\end{array}$ & $\begin{array}{l}\text { Healthy } \\
\%(\mathrm{n})\end{array}$ & $\mathrm{p}$-value* \\
\hline Boys & $60(12)$ & $50(10)$ & 0.53 \\
First born & $45(9)$ & $50(10)$ & 0.75 \\
Caesarean delivery & $15(3)$ & $0(0)$ & 0.23 \\
Furred pets & $0(0)$ & $5(1)$ & 1.00 \\
Maternal atopy & $85(17)$ & $90(18)$ & 1.00 \\
Paternal atopy & $70(14)$ & $60(12)$ & 0.51 \\
& & & \\
Breastfeeding & $100(20)$ & $100(20)$ & 1.00 \\
1 month & $25(5)$ & $35(7)$ & 0.49 \\
12 months & & & \\
Antibiotics & $15(3)$ & $30(6)$ & 0.45 \\
$1-12$ months & $50(10)$ & $30(6)$ & 0.20 \\
$12-24$ months & & & \\
& & & \\
Day-care & $0(0)$ & $5(1)$ & 1.00 \\
0-12 months & $70(14)$ & $85(17)$ & 0.45 \\
$12-24$ months & & & 0.11 \\
Probiotic group & $30(6)$ & $55(11)$ &
\end{tabular}

$X^{2}$ test. Fisher's exact test was used when the expected frequency for any cell was less than five 
TABLE II. The Shannon diversity index of the total microbiota, dominant phyla and significant genera in stool samples obtained at various ages from infants who did or did not develop atopic eczema during the first two years of life.

\begin{tabular}{|c|c|c|c|c|c|}
\hline & $\begin{array}{l}\text { Atopic } \\
\text { eczema }\end{array}$ & & Healthy & & $\begin{array}{c}\text { P- } \\
\text { value* }\end{array}$ \\
\hline & $\begin{array}{c}\mathrm{n}=20 \\
\text { median }\end{array}$ & iqr** & $\begin{array}{c}\mathrm{n}=20 \\
\text { median }\end{array}$ & iqr** & \\
\hline \multicolumn{6}{|l|}{1 week } \\
\hline Total microbiota & 1.59 & $1.33-1.77$ & 1.58 & $1.42-1.83$ & 0.78 \\
\hline Firmicutes & 0.81 & $0.48-1.27$ & 0.86 & $0.51-1.10$ & 0.53 \\
\hline Proteobacteria & 0.15 & $0.03-0.30$ & 0.32 & $0.05-0.37$ & 0.19 \\
\hline Actinobacteria & 0.29 & $0.07-0.41$ & 0.27 & $0.10-0.37$ & 0.58 \\
\hline Bacteroidetes & 0.02 & $0.00-0.51$ & 0.20 & $0.00-0.39$ & 0.60 \\
\hline \multicolumn{6}{|l|}{1 month } \\
\hline Total microbiota & 1.47 & $1.16-1.66$ & 1.69 & $1.53-2.15$ & 0.004 \\
\hline Firmicutes & 0.55 & $0.34-1.11$ & 0.61 & $0.44-0.92$ & 0.72 \\
\hline Proteobacteria & 0.15 & $0.06-0.35$ & 0.27 & $0.12-0.33$ & 0.29 \\
\hline Actinobacteria & 0.36 & $0.12-0.46$ & 0.42 & $0.20-0.67$ & 0.26 \\
\hline Bacteroidetes & 0.05 & $0.00-0.36$ & 0.48 & $0.08-0.60$ & 0.02 \\
\hline Bacteroides & 0.01 & $0.00-0.28$ & 0.44 & $0.08-0.49$ & 0.01 \\
\hline \multicolumn{6}{|l|}{12 months } \\
\hline Total microbiota & 2.90 & $2.25-3.30$ & 2.62 & $2.22-3.27$ & 0.65 \\
\hline Firmicutes & 2.31 & $1.71-2.58$ & 1.89 & $1.49-2.39$ & 0.12 \\
\hline Proteobacteria & 0.04 & $0.01-0.07$ & 0.07 & $0.04-0.13$ & 0.02 \\
\hline Actinobacteria & 0.21 & $0.11-0.41$ & 0.17 & $0.02-0.38$ & 0.43 \\
\hline Bacteroidetes & 0.16 & $0.03-0.36$ & 0.50 & $0.12-0.65$ & 0.08 \\
\hline
\end{tabular}

*Mann Whitney U-test. ** interquartile range 
TABLE III. The mean of the relative abundance of dominant phyla (bold), classes and genera (relative abundance $>1 \%$ at any age) in stool samples obtained at various ages from infants who did or did not develop atopic eczema (AE) during the first two years of life.

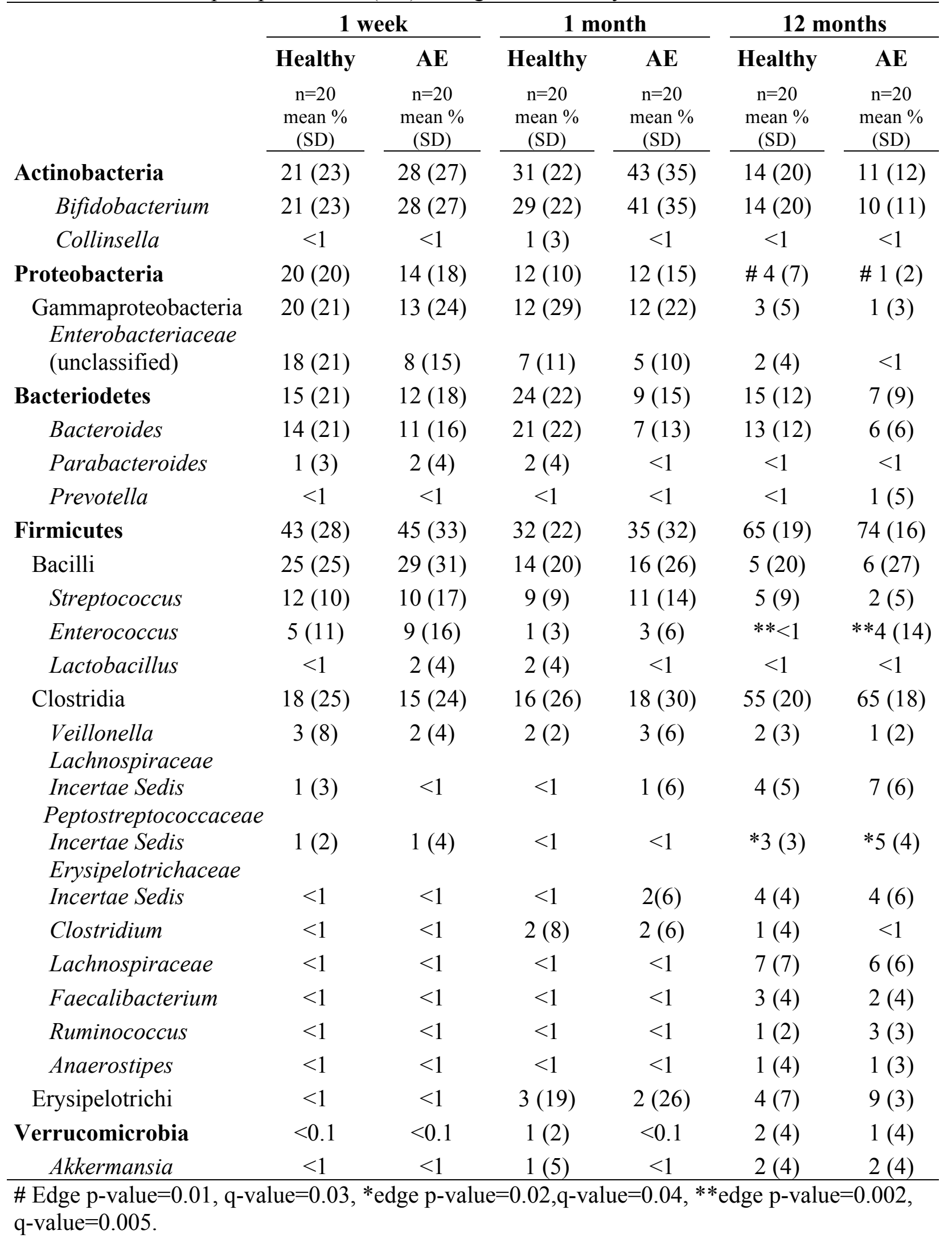

$18(25) \quad 15(24) \quad 16(26) \quad 18(30) \quad 55(20) \quad 65(18)$

\# Edge $\mathrm{p}$-value $=0$.
q-value $=0.005$. 
Abrahamsson

$478 \quad$ Legends to figures.

479

480 FIG 1.

481 Relative abundance of dominant bacterial phyla in stool samples in each subject at one week

482 (a) and at one (b) and 12 months (c) of age in 20 infants who developed atopic eczema and 20 483 infants without any allergic manifestations.

484 
(a)

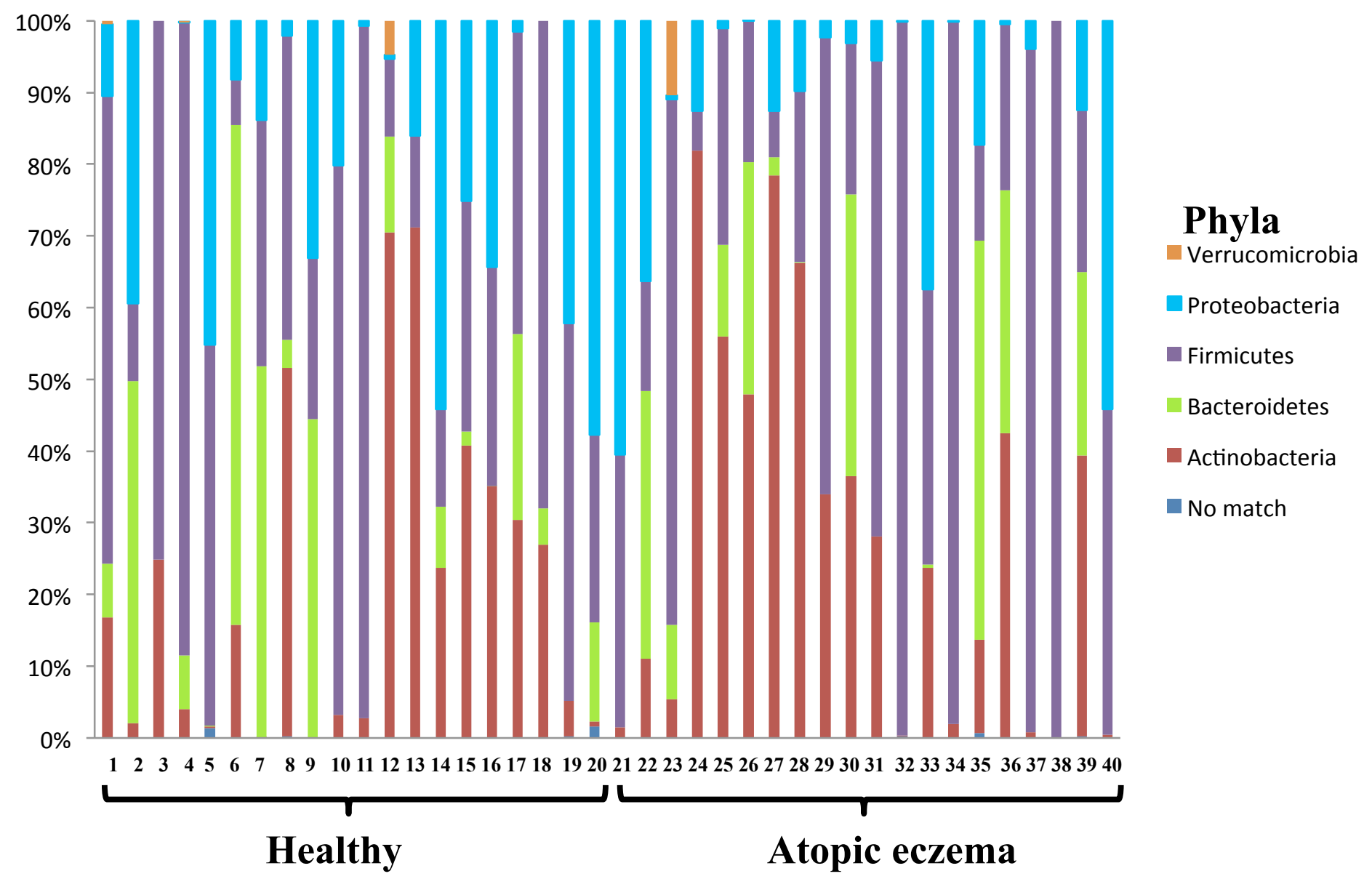


(b)

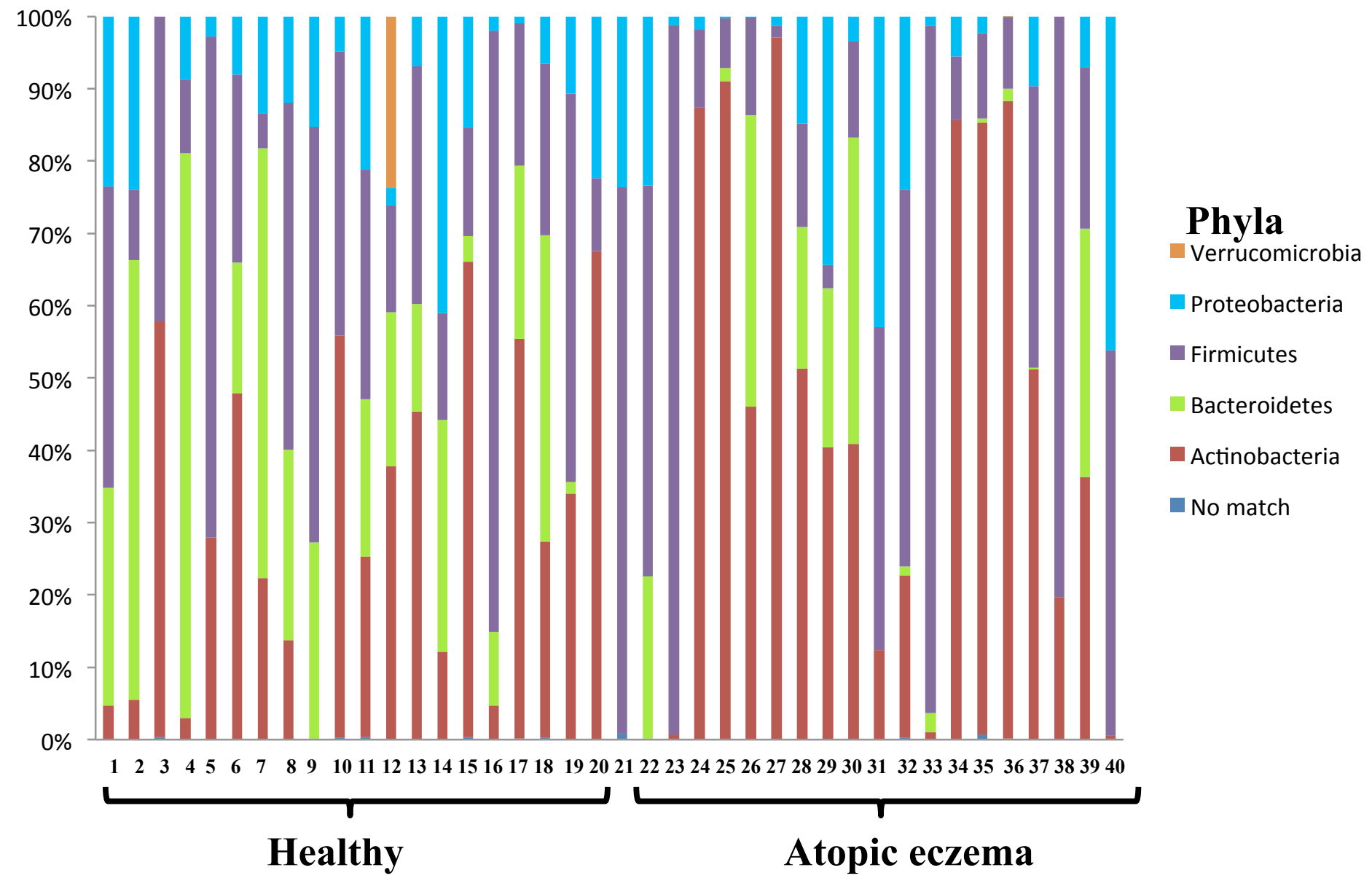


(c)

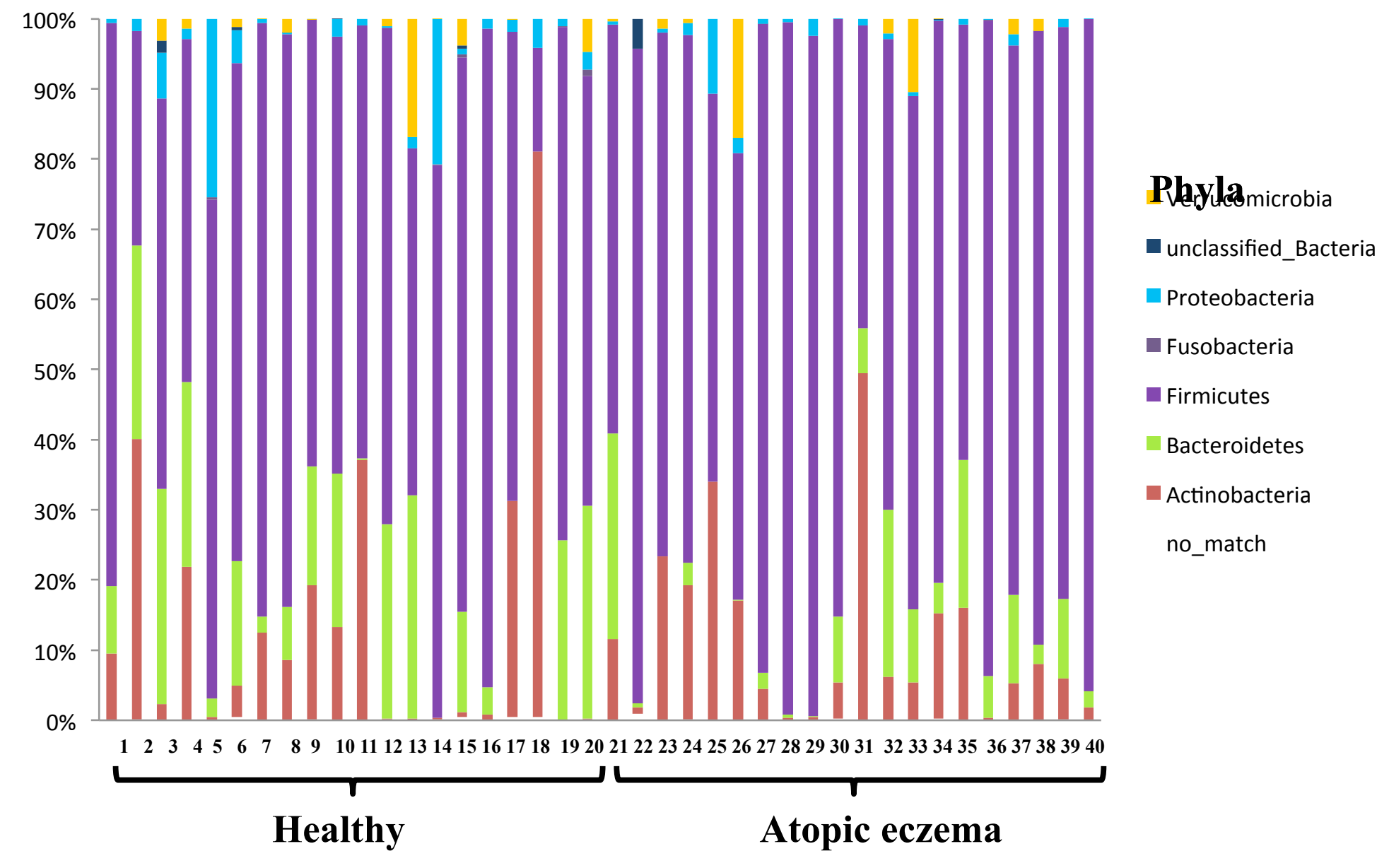

